\title{
Pregnancy Rate after Intrauterine Insemination with the Presence or Absence of Leukocytospermia in Sperms Prepared using Density Gradient Method
}

\author{
Rini Widyastuti, ${ }^{1,2,3}$ Julius Pangayoman, ${ }^{4}$ Aida Riyanti, ${ }^{5}$ Alkaustariyah Lubis, ${ }^{6}$ Mas Rizky \\ Anggun Adipurna Syamsunarno ${ }^{2,7,8,9}$

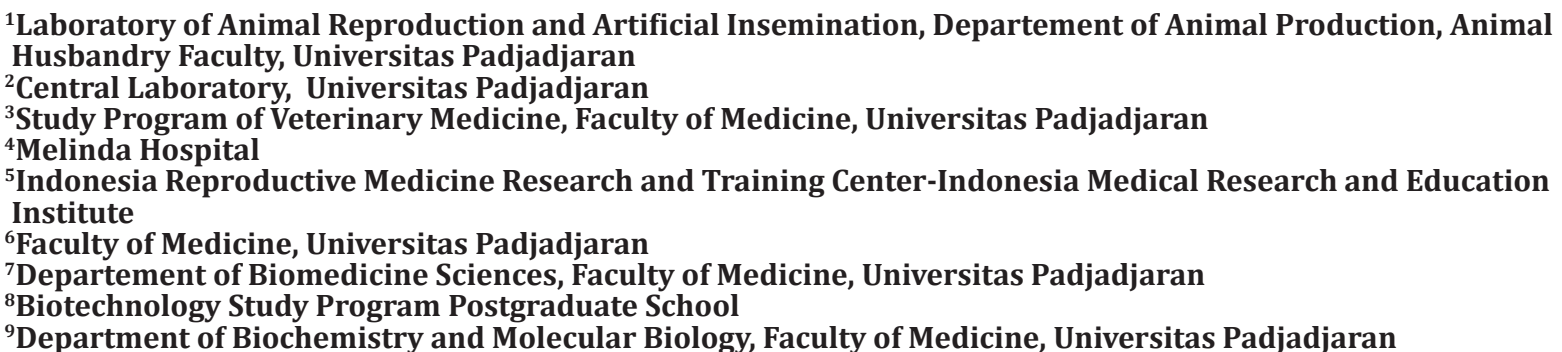

Abstract Objective: To examine the association between different concentrations of leukocyte and sperm recovery rate after sperms are prepared using density gradient method and pregnancy rate after intrauterine insemination (IUI). Increased leukocytes in semen have been associated with increased reactive oxygen species (ROS) that reduces sperm quality.

Methods: Semen samples that were collected from 31 male partners of couples undergoing infertility investigation were analyzed for sperm concentration, motility, and leucocytes concentration. Semen samples were then divided in two groups based on their leucocytes concentrations (category A: $>0$ to $<1$ $\times 10^{6} / \mathrm{mL}$; category B: $>1 \times 10^{6} / \mathrm{mL}$. Semen samples were processed using density-gradient centrifugation technique.

Results: There was a significant difference in the number of sperms harvested and sperm motility after preparation. Interestingly, pregnancy rate after IUI was higher $(\mathrm{p}<0.05)$ in non-leukocytospermia semen $(39 \%)$ when compared to leukocytospermia semen $(30 \%)$.

Received: Conclusions: Seminal leukocytes (PMNL) concentration affects pregnancy July 17, 2018 rate after intrauterine insemination.

Revised:

August 15, 2018

Keywords: Density gradient method, sperm recovery rate, intrauterine insemination, pregnancy rate

Accepted:

September 5, 2018 pISSN: 2302-1381; eISSN: 2338-4506; http://doi.org/10.15850/ijihs.v6n2.1318 IJIHS. 2018;6(2):63-6

\section{Introduction}

Urogenital tract infection (UTI) that increases the number and concentration of leucocytes up to more than $1 \times 106$ per mL, or known as leucocytospermia, is one of the most common

Correspondence:

Rini Widyastuti, Departement of Animal Production,

Animal Husbandry Faculty,

Universitas Padjadjaran

Jl. Raya Bandung-Sumedang KM 21 Jatinangor,

Indonesia

e-mail: r.widyastuti@unpad.ac.id causes of male infertility because it is associated with increased number of the reactive oxygen species (ROS) that eventually reduces sperm quality. ${ }^{1} \mathrm{~A}$ previous study has reported that a high ROS level triggers oxidative stress that impairs sperm motility, fertilization, and leads to deoxyribonucleic acid (DNA) damage. ${ }^{2}$

The techniques of sperm preparation prior to intrauterine insemination (IUI) play an important role in removing the leucocytes, seminal plasma, and also debris from the sperm as well as increasing the concentrations 
of motile sperm prior to introduction into uterine cavity which will eventually increase the chances of conception. The techniques applied vary from laboratory to laboratory and even from patient to patient. In addition, these techniques are also very useful to treat infertility in male individuals by improving sperm motility and the pregnancy rates after IUI. In artificial reproductive technology (ART) there are 4 techniques for sperm preparation i.e. simple washing, swim-up, density gradient, and mini gradient. ${ }^{3}$

Compared to the Swim-up method, better sperm preparations using Pure Sperm Density gradient method is able to better improve the quality of the sperms. ${ }^{4}$ When using density gradient method, it is showed that immotile or abnormal sperm cells, leucocytes, and cell debris are thoroughly cleared from the final solution, decreases ROS leak and eventually decreases the DNA damage. ${ }^{5}$ Furthermmore, this method is more effective because it is easy to perform and has fewer critical points that are sensitive to errors during preparation. The link between leukocytospermia, semen quality, and pregnancy rate after intrauterine insemination using specific sperm preparation method is inconsistent. The study presented here was designed to evaluate the effects of leukoscytospermia on the sperm recovery rate after sperm preparation using density gradient method and pregnancy rate outcomes after IUI.

\section{Methods}

The study involved 36 couples who underwent IUI because of male infertility factor including oligo, astheno, and also terratozoospermia in Melinda Hospital, Bandung, Indonesia. Semen specimens were collected by masturbation after 2-7 days of sexual abstinence. Semen specimens collected were divided into two groups according to the amount of leucocytes present in the semen. Specimen in group 1 had WHO-defined leukeocytospermia of $>1 \mathrm{x}$ $10^{6} / \mathrm{mL}(\mathrm{n}=23)$ and non-leukocytospermia in group 2 had seminal leucocytes less than $1 \mathrm{x}$ $10^{6} / \mathrm{mL}(\mathrm{n}=23)$.

Semen analysis was conducted during the period of 30-60 minutes after liquefaction based on a guideline published by the World Health Organization (2010) through the use of a phase contrast microscope (Nikon E-400, Japan). Semen liquefaction time, appearance, volume, $\mathrm{pH}$, and viscosity were determined. These specimens were analyzed for sperm concentration as well as progressive motilities before and after sperm preparation.

All samples underwent density gradient and prepared spermatozoa were processed using discontinuous density gradient centrifugation. One milliliter of a $90 \%$ density lower layer, 1 $\mathrm{mL}$ of a $45 \%$ density upper layer, and $1 \mathrm{~mL}$ of semen (1/1/1) were pipetted and put into a 15 $\mathrm{mL}$ conical Falcon tube. The mixture was then centrifuged at 1,500 rpm for 10 minutes. After centrifugation, the supernatant was removed and the spermatozoa (pellet) was placed into another 15-mL Falcon tube containing $5 \mathrm{~mL}$ of modified-earle's buffer salt solution (mEBSS) supplemented with 3\% Human Albumin Serum (HAS, Vitrolife). The solution of spermatozoa pellet was then centrifuged at $1500 \mathrm{rpm}$ for 10 minutes. The final pellet was re-suspended in the same medium solution to obtain a final volume of $0.5 \mathrm{~mL}$. A $10-\mu \mathrm{L}$ aliquot was used to perform the analysis of post-processing seminal parameters. The harvested quality sperms were suspended in the final volume of $0.3 \mathrm{~mL}$ of sperm preparation medium to be used for IUI. All semen samples were analyzed for sperm motility and sperm concentration in the same person, in the same laboratory, and in the same condition.

Recombinant FSH was used for stimulating ovarium. Female patients were scheduled for the IUI procedure when at least one follicle that was $20 \mathrm{~mm}$ in size was detected through ultrasound scanning. Insemination using the prepared sperms was performed with a sterile catheter and $1 \mathrm{~mL}$ syringe. Here, the catheter was gently passed through the cervical canal and $0.3 \mathrm{~mL}$ of the sperm suspension was expelled slowly into the uterine cavity close to the tubal junction. The female patients were asked to rest in lithotomy position for 30 minutes after IUI. The pregnancy test was performed 14 days after insemination.

Statistical analysis was conducted using SPSS software version 16 (SPSS Inc, Chicago, IL, USA). All data were analyzed using Student's T-test. The level of significance different was $\mathrm{p}<0.05$.

\section{Results}

Sperm washing must be performed to prepare the sample for insemination in IUI cycle. The baseline data of sperm characteristic before preparation was used to choose the method of preparation and predict sperm recovery rate after the process. The baseline data of sperm characteristic in this study consisted 
of the number and motility of the sperms. The average number of sperms from sample without leukocytospermia was significantly lower when compared to sperm samples that presented with leucocytospermia $(\mathrm{p}<0.05)$ $(69.60 \pm 5.92$ versus $84.78 \pm 5.75)$. Interestingly, the sperm motilities in both groups were not significantly different $(\mathrm{p}>0.05) \quad(35.39 \pm 10.54$ vs $38.69 \pm 2.60$ ).

The number of sperms harvested as well as the total number of motile sperms in the samples with and without leukocytospermia are shown in this study (Table). The results showed that the presence of leukocytes in the semen significantly increased the number of sperm harvested and a total number of motile sperms $(p<0.05)$. However, the sperm motility after performing density gradient method was not significantly different in both groups $(\mathrm{p}<0.05)$. Interestingly, pregnancy rate after IUI, in female inseminated with semen without leukocytospermia yielded the higher pregnancy rate $39 \%)(\mathrm{p}<0.05)$ compared to semen with leukocytospermia (30\%).

\section{Discussion}

The success rate of IUI depends on factors such as drugs used for stimulation, triggering time, number of cycles and the total number of motile sperms after washing. A previous study suggested that the number of sperms inserted into uterus will decrease when the number of washed motile sperm is insufficient. ${ }^{6}$ Sperm motility is an important factor in IUI because it is an indicator of sperm ability to reach and fertilize the ovulated oocyte. It is reported that the association between leukocyte and sperm quality will depend on concentration with motility percentage increases when the leukocyte concentration is 0 to $<1.0 \times 10^{6} / \mathrm{mL}$ and decreases when leukocyte concentration is $>1.0 \times 106.7$ This fact is contrary to the results of a previous study reported that there was no significant difference between no leukocytes, low-level leukocytes, and leukocytospermia groups in baseline clinical characteristics and conventional parameters that includes semen concentration, motility, and morphology). ${ }^{8}$

Leukocyte, bacteria, and dead spermatozoa produce reactive oxygen species or ROS that negatively influence the ability of sperms to fertilize the egg. ${ }^{9}$ It was reported that patient with low seminal leukocytes concentration $(<1.0 \times 106 / \mathrm{mL})$ had significantly higher level of ROS and sperm DNA damage and there was no significant difference in ROS level between low leukocytes level and leukocytospermia. ${ }^{10}$

A significant positive correlation between leukocytospermia and the oxidative stress has been reported, suggesting that the leukocytes are the primary source of ROS in semen. ${ }^{11,12}$ Although the primary source of ROS is WBCs, a portion of these ROS is produced by sperms. The hydroxyl radical formed through HaberWeiss reaction will attack unsaturated fatty acids in sperm membrane and initiate lipid peroxidation. ${ }^{12,13}$ Catalase as well as superoxide dismutase are antioxidant enzymes. It is also reported that a positive correlation is found between sperm concentration and superoxide dismutase and a negative correlation is seen with leukocytospermia. ${ }^{14}$

Group with leukocytospermia in this study presented a lower pregnancy rate due to PNML concentration was higher than physiological condition which triggers a higher level of ROS production. ROS has a correlation with the ability of sperms to fertilize mature oocytes; including two primary factors, a slow rate of forwarding progression of sperm and the presence of excess numbers of white cells in the semen..$^{15}$ The recent study showed the same result as the result of a previous study by AlDujaily reported that the pregnancy rate after

Table Comparison of Sperm Parameter after Performed Density Gradient Method and Pregnancy Rate after IUI on Semen with and without Leukocytospermia

\begin{tabular}{|c|c|c|}
\hline Parameter & $\begin{array}{l}\text { PMNL Concentration } \\
>0<1 \times 10^{6} / \mathrm{mL}\end{array}$ & $\begin{array}{c}\text { PMNL concentration } \\
>1 \times 10^{6} / \mathrm{mL}\end{array}$ \\
\hline Total harvested sperm (millions) & $2.22 \pm 0.80^{\mathrm{a}}$ & $2.52 \pm 0.98^{b}$ \\
\hline Total motile sperms (millions) & $1.95 \pm 0.67^{\mathrm{a}}$ & $2.23 \pm 0.84^{\mathrm{b}}$ \\
\hline Sperm motility (\%) & $88.61 \pm 6.48^{\mathrm{a}}$ & $89.04 \pm 5.77^{\mathrm{a}}$ \\
\hline Pregnancy rate (\%) & $39.50 \pm 0.50^{a}$ & $30.43 \pm 4.70^{\mathrm{b}}$ \\
\hline
\end{tabular}

Note: Different superscripts in the same row indicate a statistically significant difference $(\mathrm{p}<0.05)$. 
IUI in leukocytospermic couples was lower compared to normozoospermic couples. The percentage was $20 \%$ in normozoospermic and $13.3 \%$ in leukocytospermia. ${ }^{16}$ These findings indicate that leukocytic infiltration into male reproductive tract is only harmful when the contamination level of leucocyte is high enough $(>1 \times 106 / \mathrm{mL})$ to overwhelm the antioxidants present in human seminal plasma. The high levels of leucocyte contamination will disrupt

\section{References}

1. Cooper TG, Noonan E, von Eckardstein S, Auger J, Baker HW, Behre HM, et al. World Health Organization reference values for human semen characteristics. Hum Reprod Update. 2010;16(3):231-45.

2. Aitken RJ, Koppers AJ. Apoptosis and DNA damage in human spermatozoa. Asian J Androl. 2011;13(1):36-42.

3. Henkel R. Sperm preparation: state-of-theart-physiological aspects and application of advanced sperm preparation methods. Asian J Androl. 2012;14(2):260-9.

4. Amiri I, Ghorbani M, Heshmati S. Comparison of the DNA fragmentation and the sperm parameters after processing by the density gradient and the swim up methods. J Clin Diagn Res. 2012;6(9):1451-3.

5. Shamsi MB, Kumar R, Dada R. Evaluation of nuclear DNA damage in human spermatozoa in men opting for assisted reproduction. Indian J Med Res. 2008;127(2):115-23.

6. Dorjpurev U, Kuwahara A, Yano Y, Taniguchi T, Yamamoto Y, Suto A, et al. Effect of semen characteristics on pregnancy rate following intrauterine insemination. J Med Investigation. 2011;58(1):127-33.

7. Lackner JE, Agarwal A, Mahfouz R, du Plessis SS, Schatzl G. The association between leukocytes and sperm quality is concentration dependent. Reprod Biol Endocrinol. 2010;8(1):12.

8. Agarwal A, Mulgund A, Alshahrani S, Assidi M, Abuzenadah AM, Sharma R, et al. Reactive oxygen species and sperm DNA damage in infertile men presenting with low levekl leukocytospermia. Reprod Biol Endocrinol [serial on the internet]. 2014 Dec [cited 2018 jan 8];12(126):[about 8p.]. Available from:https://rbej.biomedcentral.com / articles/10.1186/1477-7827-12-126.

9. Domes T, Lo KC, Grober ED, Mullen JB, Mazzulli T, Jarvi K. The incidence and effect the fertility in vivo.

The results indicate that leukocytospermia may not have a negative effect on the sperm preparation outcome though it might affect pregnancy rate after Intrauterine Insemination procedure. The absense of sperm morphology analysis as well as the retrospective nature of the study create limitations in this study. A future prospective study on more patients with sperm morphology assessment is needed.

of bacteriospermia and elevated seminal leukocytes on semen parameters. Fertil Steril. 2012:97(5):1050-5.

10. Alshahrani S, Agarwal A, Assidi M, Abuzenadah AM, Ayaz A, Sharma R. The effect of low level leukocytospermia on oxidative stress markers in infertile men. BMC Genomics. [serial on the internet]. 2014 Apr [cited 2018 jan 8];15(Suppl 2):[about 2p.]. Available from: https://www.ncbi.nlm.nih.gov/pmc/articles/ PMC4075643/.

11. Aggarwal R, Puri M, Dada R, Saurabh G. Correlation between leukocytospermia and pxidative stress in male partners of infertile couples with leukocytospermia. Int J Reprod Contracept Obstet Gynecol. 2017;4(1):168-72

12. Sandoval JS, Raburn D, Muasher S. Leukocytospermia: overview of diagnosis, implications, and management of a controversial finding. Middle East Fertil Soc J. 2013;18(3):129-34

13. Aitken RJ, Curry BJ. Redox regulation of human sperm function: from the physiological control of sperm capacitation to the etiology of infertility and DNA damage in the germ line. Antioxid. Redox Signal. 2011;14(3):367-81.

14. Pasqualotto FF, Umezu FM, Salvador M, Borges E, Sobreiro P, Pasqualotto B. Effect of cigarette smoking on antioxidant levels and presence of leukocytospermiain infertile men:a prospective study. Fertil Steril. 2008;90(2):278-83.

15. Barraud-Lange V., Pont JC, Pocate K, Kunstmann JM, Chalas-Boissonas C, Ducot B, Wolf JP. Seminal leukocytes and clinical outcomes with donor sperm insemination. Fertil. Steril. 2011;96(6):1320-4

16. Al-Dujaily SS, Al-Jnabi $\mathrm{MH}$, Jasim SN. The influence of leukocytospermia and teratozoospermia in IUI outcome. IJAR. 2015;3(11):255-65. 